\title{
Blauer Laser in der Akupunktur - ein erster Review-Report
}

\author{
Gerhard Litscher $^{\mathrm{a}}$ Lu Wang $^{\mathrm{a}}$ Zheng Xie $^{\mathrm{a}, \mathrm{b}}$ Ingrid Gaischek ${ }^{\mathrm{a}}$ \\ ${ }^{a}$ Forschungseinheit für biomedizinische Technik in Anästhesie und Intensivmedizin und TCM Forschungszentrum Graz, \\ Medizinische Universität Graz, Österreich \\ ${ }^{\mathrm{b}}$ Beijing University of Chinese Medicine, Peking, China
}

\section{Schlüsselwörter}

Akupunktur · Blaue Laserakupunktur · Blaue Lasernadel .

Mikrozirkulation $\cdot$ Herzrate

\section{Zusammenfassung}

In der Akupunktur haben in den vergangenen 15 Jahren kleine, handliche Laser Einzug gehalten, mit denen Akupunkturpunkte bestrahlt werden, deren Effekte und therapeutische Wirkung aber in naturwissenschaftlichen Untersuchungen nach wie vor nicht eindeutig belegt sind. Die schmerzfreie, nichtinvasive Laserakupunktur mit optischen Nadeln (infrarot + roter Laser) kann hingegen im Gehirn und in der Peripherie spezifische, reproduzierbare Veränderungen induzieren. Dies äussert sich in Verschiebungen von unterschiedlichen Parametern, wie beispielsweise der Blutströmungsgeschwindigkeit, und kann erstmals mit Methoden der High-Tech Akupunktur ${ }^{\oplus}$ objektiviert werden. Ziel des vorliegenden Review-Reports ist es, die bislang vorliegenden experimentellen Messungen zur Laserstimulation mit einem blauen Laser zusammenzufassen. Diese blaue Lasernadelstrahlung ( $405 \mathrm{~nm}$ ) erzeugt - wie in Grazer Pilotstudien festgestellt wurde - spezifische Effekte, die sich unter anderem in einem nadelähnlichen De-Qi-Gefühl äussern können. Mit dem neuen Verfahren könnte sich eine sinnvolle Ergänzung zur Nadelakupunktur für Kinder oder PatientInnen mit Nadelangst ergeben, wobei die klinischen Anwendungen in Bezug auf die blaue Laserakupunktur erst ausgelotet werden müssen.

\section{Einleitung}

Laserlicht spielt sowohl im Rahmen der Phototherapie als auch der Akupunktur in den letzten Jahren eine sehr wichtige Rolle. In zahlreichen wissenschaftlichen Arbeiten konnte gezeigt werden, dass der Laser biologisch wirksame Effekte hervorrufen kann [1]. Auch die Akupunktur wurde in den letzten Jahrzehnten vor allem im Westen entscheidend durch neue Techniken, wie beispielsweise die Laserakupunktur, geprägt. Während Akupunktur mit roten und/oder nahinfraroten Lasernadeln (optische Applikatoren zur kontinuierlichen und multipunktuellen Laserstimulation) bereits zum Standard innovativer Akupunkturbehandlungen gehört [2], hat der «blaue Laser»

\section{Keywords}

Acupuncture · Blue laser acupuncture · Blue laser needle . Microcirculation $\cdot$ Heart rate

\section{Summary}

Blue Laser in Acupuncture - a Preliminary Review Report

Over the last 15 years, small, handheld lasers have been used in acupuncture. With these it is possible to stimulate acupuncture points, however, their effects and therapeutical effectiveness are still discussed controversially in scientific investigations. In contrast, the painless, noninvasive laser acupuncture using optical needles (infrared + red laser) can induce specific, reproducible changes in the brain and in the periphery. This can be demonstrated by changes in different parameters, e.g. blood flow velocity, and can be quantified using high-tech acupuncture $^{\oplus}$ methods for the first time. It is the goal of the present review report to summarize the currently available experimental measurements concerning blue laser stimulation. Blue laser needle radiation ( $405 \mathrm{~nm}$ ) induces specific effects, among others deqi sensations similar to those of needle acupuncture. This was shown in pilot studies performed in Graz. The new method could be a sensible supplement to needle acupuncture in children or patients with needle phobia; the clinical applications for the blue laser acupuncture, however, have to be explored first.

bislang in erster Linie im Bereich der Wundheilung, bei dermatologischen Erkrankungen und im Bereich der intravasalen Blutbestrahlung seine Hauptanwendung gefunden $[3,4]$.

Im Bereich der Akupunktur wurde der blaue Laser bislang ausschliesslich von unserer Forschungsgruppe eingesetzt. Im Rahmen eines kooperativen Forschungsprojektes zwischen Österreich und China wurden federführend von unserem Team in Korea und den USA die ersten wissenschaftlichen Resultate zu dieser neuen Methode, der «Blauen Laserakupunktur», publiziert [5-7]. Inhalt dieses kurzen «Review-Reports» ist eine Zusammenfassung der bislang erforschten akupunkturspezifischen Effekte des blauen Lasers in komprimierter Form.

$\begin{array}{ll}\text { KARGER } & \odot 2010 \text { S. Karger GmbH, Freiburg } \\ \begin{array}{ll}\text { Fax }+497614520714 & \text { Accessible online at: } \\ \text { Information@Karger.de } \\ \text { www.karger.com }\end{array} & \text { www.karger.com/szg }\end{array}$

Univ.-Prof. DI Dr. techn. Dr. scient. med. Gerhard Litscher

Forschungseinheit für biomedizinische Technik in Anästhesie und Intensivmedizin und TCM Forschungszentrum Graz, Medizinische Universität Graz

Auenbruggerplatz 29, $8036 \mathrm{Graz}$, Österreich

Tel +43316 385-13907/-83907, Fax -13908

gerhard.litscher@medunigraz.at 


\section{Methoden}

Blaue Laserakupunktur

Zur Akupunktur wurde ein Lasermicroneedle ${ }^{\circ}$-System mit HalbleiterLaserdioden (Laserneedle EG GmbH, Wehrden, Deutschland) verwendet (Abb. 1). Im System sind Laser mit blauer Emission (Wellenlänge $405 \mathrm{~nm}$ ) integriert. Die Leistung der Lasernadel betrug $110 \mathrm{~mW}$ und der Durchmesser $500 \mu \mathrm{m}$; die Stimulationsdauer war mit 10 min festgelegt.

\section{Studienteilnehmer}

Die demografischen Daten der gesunden ProbandInnen sind Tabelle 1 zu entnehmen. Insgesamt wurden im Rahmen der beiden Pilotstudien 40 Personen untersucht. Keine/r der TeilnehmerInnen stand unter dem Einfluss einer Medikation und hatte neurologische oder kardiologische Auffälligkeiten. Die ProbandInnen waren über die Untersuchungen so weit, wie es das Studiendesign zuliess, aufgeklärt. Sie wurden für den Aufwand der Untersuchungen remuneriert. Die Studien wurden von der lokalen Ethikkommission genehmigt, und alle TeilnehmerInnen gaben eine schriftliche Einverständniserklärung ab.

\section{Akupunktur und Prozedere}

Im Rahmen der ersten Akupunkturstudie mit dem blauen Laser wurden mögliche Temperatur- und Mikrozirkulationsveränderungen nach Stimulation des Akupunkturpunktes Hegu (Di.4) objektiviert [5]. Dabei wurde das Hautareal um den genannten Akupunkturpunkt in einer Entfernung von $1 \mathrm{~mm}$ näher untersucht. Gleichzeitig wurde der Bereich der Fingerspitze des Zeigefingers vor, während und nach kontinuierlicher Stimulation überwacht. Zur Anwendung gelangten eine Infrarot-Wärmebildkamera (FLIR ${ }^{\circ}$ i5; Flir Systems Inc., Portland, OR, USA) und ein Laser-DopplerSystem (DRT 4; Moor Instruments Ltd., Devon, UK) (Messprozedere vergleiche Abb. 2A).

Bei der zweiten Studie wurde Akupunktur am Punkt Neiguan (KS.6) beidseits durchgeführt. Die blaue Lasernadel wurde mit einem speziellen Klebeadapter auf die Haut aufgeklebt, aber nicht eingestochen. Diese Unter- suchung bestand aus zwei Teilen: einer aktiven Stimulation (Abb. 2B) und einer sog. Placebomessung (Abb. 2C), bei der der Laser inaktiv war.

Die Versuchspersonen, bei denen die beiden Punkte (KS.6 Neiguan) stimuliert wurden, wurden mit einem medilog ${ }^{\circledR}$ AR12 System (Huntleigh Healthcare, Cardiff, UK) elektrokardiografisch (EKG) überwacht. Das System tastet das EKG mit einer Rate von $4096 \mathrm{~Hz}$ ab und ermöglicht so eine zuverlässige Detektion der RR-Intervalle. Die Daten wurden mit spezieller Software (Darwin HRV; Huntleigh Healthcare, Cardiff, UK) analysiert.

\section{Statistische Analyse}

Der mikrozirkulatorische Parameter Flux (= Produkt aus Konzentration $\times$ Geschwindigkeit der Erythrozyten) sowie die Temperatur und die Herzrate werden als Box Plots grafisch präsentiert («paired t-test» bzw. «one-way repeated measures ANOVA on ranks» und «Tukey-Test»; SigmaPlot 11.0; Systat Software, Chicago, IL, USA). Das Signifikanzniveau wurde mit $\mathrm{p}<0,05$ festgelegt.

\section{Ergebnisse}

Über 90\% der StudienteilnehmerInnen nahmen die Stimulation durch den blauen Laser wahr und gaben an, ein plötzliches sog. De-Qi-Gefühl zu verspüren.

Im Bereich des Punktes Di.4 (Hegu) kam es zu einem signifikanten Anstieg der Temperatur $(\mathrm{p} \leq 0,001)$ und des Flux ( $\leq 50001)$ während der Stimulation mit dem blauen Laser (Abb. 3, 4).

Auch 5 min nach Akupunktur war der Flux an der Messstelle (1 mm von Di.4 entfernt, vergleiche Abb. 3) noch signifikant erhöht. An der Fingerspitze des Zeigefingers

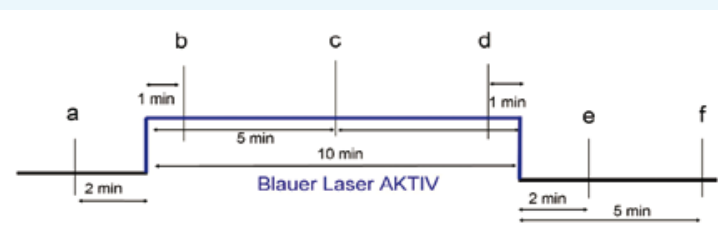

A

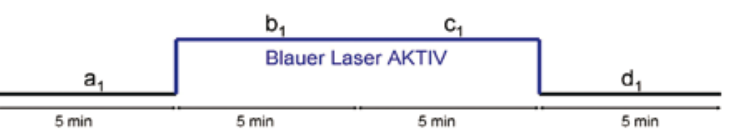

B

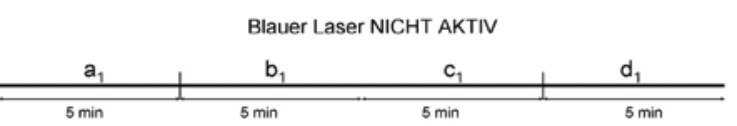

C
Abb. 1. Lasersystem für die Durchführung der ersten Studien zur blauen Laserakupunktur im TCM Forschungszentrum an der Medizinischen Universität Graz.

Tab. 1. Demografische Daten der bislang mit dem blauen Laser untersuchten Versuchspersonen

\begin{tabular}{llll}
\hline & Gesamt & Di.4 (Hegu) & KS.6 (Neiguan) \\
\hline Anzahl der Studienteilnehmer & 40 & 15 & 25 \\
Frauen & 26 & 11 & 15 \\
Männer & 14 & 4 & 10 \\
Alter & & & \\
Mittelwert & 29,5 & 29,6 & 29,4 \\
Standardabweichung (SD) & 7,0 & 8,1 & 6,6 \\
Bereich & $22-50$ & $22-50$ & $23-50$ \\
\hline
\end{tabular}


konnten gleichzeitig keine signifikanten Veränderungen registriert werden.

Abbildung 5 fasst die Ergebnisse der Herzrate der 25 ProbandInnen zusammen. Signifikante Veränderungen konnten unmittelbar nach Beginn und während der blauen Laserstimulation (Akupunkturpunkt KS.6 Neiguan) festgestellt werden. Dieser Effekt war anhaltend und auch nach der Akupunktur noch manifest. Bei der Placebomessung war dies nicht in gleichem Ausmass der Fall, wenngleich
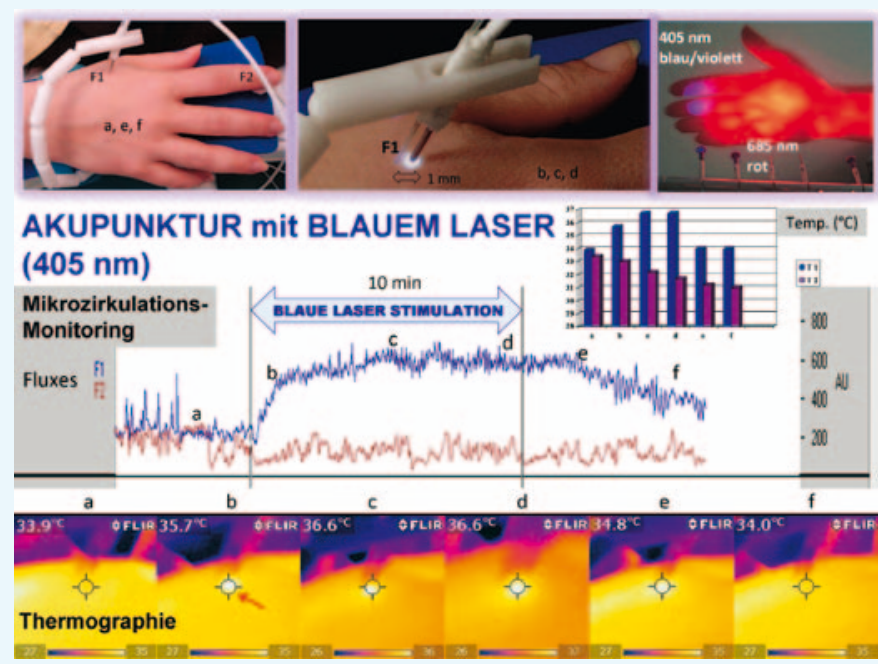

Abb. 3. Erster Einsatz der blauen Laserakupunktur mit einer Wellenlänge von $405 \mathrm{~nm}$. Man beachte den signifikanten Anstieg der Mikrozirkulation (Flux1, F1; blaue Kurve) $1 \mathrm{~mm}$ neben der blauen Lasernadel am Akupunkturpunkt Hegu (Di.4). Die Temperatur (T1) wurde an dieser Stelle mittels Wärmebildkamera gemessen. Man beachte weiterhin den Temperaturanstieg von $33,9^{\circ} \mathrm{C}$ auf $36,6^{\circ} \mathrm{C}$ in der ROI («region of interest»). Die Registrierung von Flux2 (F2) und Temperatur (T2) erfolgte am Zeigefinger (modifiziert aus [5]). sich eine in der Signifikanz nicht so ausgeprägte Abnahme gegen Ende der Registrierung fand.

\section{Diskussion}

Der blaue Laser wurde im Rahmen der Akupunkturforschung an unserer Institution weltweit erstmals wissenschaftlich untersucht. Das Grundprinzip der blauen LaserMethode kommt dabei aus der Informationstechnologie. Die Blu-ray Disc ist beispielsweise ein darauf basierendes Speichermedium der neuesten Generation. Wenngleich die Kosten eines blauen Lasers derzeit noch das circa Zehnfache eines roten Lasers betragen, so kann man mit blau-violetten Wellenlängen in einem Bereich von $405 \mathrm{~nm}$ und einem speziell fokussierten Strahl trotz geringer Eindringtiefe deutliche reproduzierbare Effekte, wie etwa Steigerungen der Durchblutung in kleinsten Gefässen, während und nach einer Akupunktur erzielen [5]. Auch die Auslösung eines für die Nadelakupunktur typischen De-Qi-Gefühls, welches sich oftmals als leichtes elektrisches Kribbeln äussert, ist mit dem neuen blauen Laser zumindest in ähnlicher Weise möglich [5-7]. Genau hier setzen auch die wissenschaftlichen Untersuchungen an. Für ChinesInnen ist das Empfinden dieses De-Qi-Gefühls bei der Akupunktur eine wichtige Prämisse, um eine erfolgreiche Behandlung durchzuführen. Während man unter der Verwendung eines roten oder infraroten Lasers (z.B. $658 \mathrm{~nm}$ und $785 \mathrm{~nm}$ ) diesen Reiz nicht sofort spürt [8], ist dies hingegen beim blauen/violetten Laser ( $405 \mathrm{~nm}$ ) anders. Mehr als 90\% der untersuchten erwachsenen ChinesInnen und auch EuropäerInnen berichten von einem
Abb. 4. Veränderungen von Flux und Temperatur, gemessen $1 \mathrm{~mm}$ neben Hegu (Di.4; Flux1, Temp1) und an der Spitze des Zeigefingers (Flux2, Temp2). Man beachte den signifikanten Anstieg der Mikrozirkulation (Flux1) während Akupunkturstimulation (vergleiche Abb. 3). Die horizontale Linie in der Box gibt die Lage des Medians an; die Enden der Boxen definieren die 25ste und 75ste Perzentile, und die Fehlerbalken definieren die 10te und 90ste Perzentile. Die Punkte sind einzelne Messwerte ausserhalb der Fehlerbalken (modifiziert aus [5]).
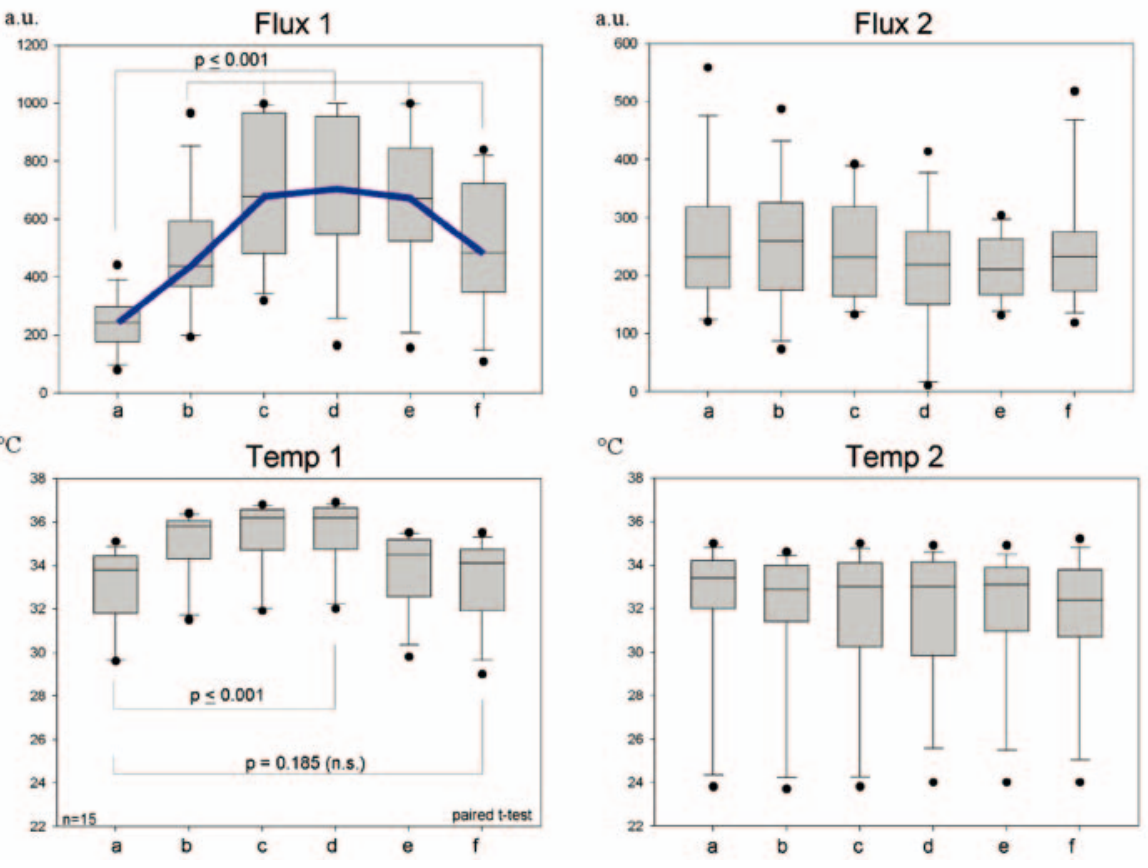


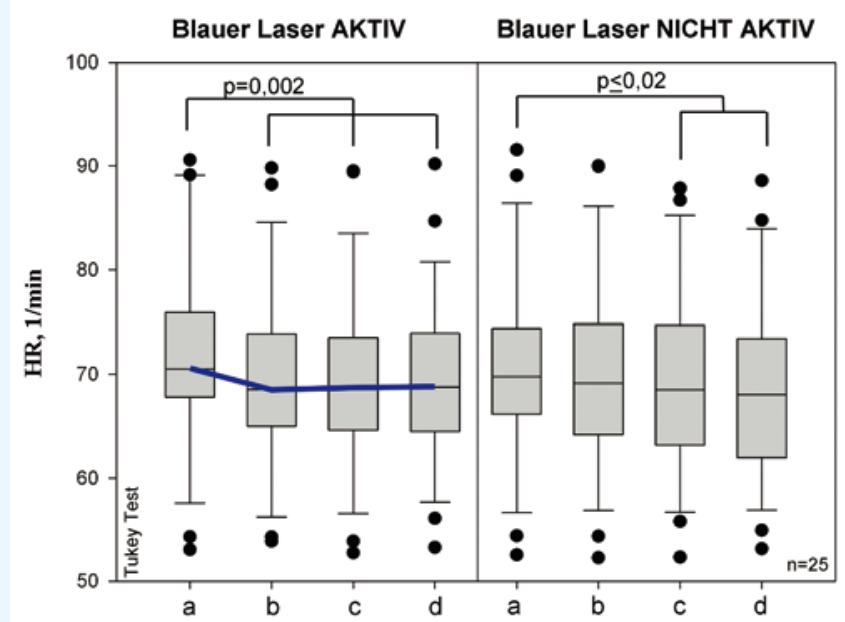

Abb. 5. Box-Plot-Darstellung der Herzrate (HR) während der beiden Bedingungen «Blauer Laser aktiv» (linke Seite) und «Blauer Laser nicht aktiv» (rechte Seite). Man beachte das signifikante Absinken der HR während blauer Laserstimulation am Punkt Neiguan (KS.6). Messphasen vergleiche Abb. 2; weitere Details siehe auch Abb. 4.

sofortigen, tief empfundenen Reiz, ähnlich einem De-QiGefühl mit einer Metallnadel. «Der Effekt ist vergleichsweise nicht ganz so stark wie ein Ameisenbiss, jedoch länger anhaltend», berichtet beispielsweise eine chinesische Probandin. Wurde der Akupunkturpunkt Neiguan stimuliert, so kam es zu einem signifikanten Absinken der Herzrate in Verbindung mit einem angenehmen, stressreduzierenden Gefühl. Diesem speziellen Punkt, der auch als «Perikard 6» oder «Kreislauf 6» bezeichnet wird und der sich im Bereich der Handgelenksfalte befindet, sagt man nach Vorstellungen der traditionellen chinesischen Medizin (TCM) nach, dass er eine kreislaufregulierende Wirkung erzielen kann. Die Effekte traten in einer Kontrolluntersuchung, bei der der blaue Laser deaktiviert war, nicht auf.
Zum blauen Laser gibt es generell bislang noch sehr wenige Studien im Bereich der Medizin. Neben Forschungen auf zellulärer Ebene in Shanghai [9] beschäftigen sich auch Wissenschafter in New York [10], Finnland [1], Deutschland [4] und Japan [11, 12] mit der Thematik. Der Ansatz, den blauen Laser in der Akupunktur zu verwenden, ist neu und liefert offensichtlich trotz geringer Eindringtiefe (blau: 1-2 mm vs. rot und infrarot: $2-3 \mathrm{~cm}$ ) Effekte, die noch näher untersucht werden sollten [5-7].

\section{Schlussfolgerung}

In Zukunft könnte das Verfahren der blauen Laserakupunktur eventuell bei PatientInnen mit Bluthochdruck oder generell bei altersbedingten Erkrankungen des HerzKreislauf-Systems als schonende, regulative Methode additiv eingesetzt werden. Vorher sind allerdings noch zahlreiche Grundlagenstudien durchzuführen.

\section{Dank}

Dieser Review-Artikel basiert auf zwei englischsprachigen Originalpublikationen der TCM Forschungsgruppe Graz, die 2009 publiziert wurden [5, 6]. Die Forschungen wurden im Rahmen der Projekte «Bioengineering and Clinical Assessment of High-Tech Acupuncture: A Sino-Austrian Research Pilot Study» (unterstützt vom Bundesministerium für Wissenschaft und Forschung (BMWF), dem Bundesministerium für Gesundheit (BMG) und dem Eurasia Pacific Uninet) sowie «Computergestützte Analyse der Herzratenvariabilität während unterschiedlicher Akupunkturmethoden» (Projekt Nr. 13463, Jubiläumsfonds der Österreichischen Nationalbank) durchgeführt.

Die Autoren danken dem Wissenschaftsressort der Stadt Graz, das das Projekt «Blauer Laser in der Akupunktur» unterstützt.

\section{Disclosure Statement}

There is no conflict of interest to declare.

\section{Literatur}

1 Karu T: Ten lectures on basic science of laser phototherapy. Grängesberg, Prima Books, 2007.

2 Litscher G, Schikora D (eds): Lasernadel-Akupunktur. Wissenschaft und Praxis. Lengerich, Pabst Science, 2004.

3 Mittermayr R, Osipov A, Piskernik C, Haindl S, Dungel P, Weber C, Vladimirov YA, Redl $\mathrm{H}$, Kozlov AV: Blue laser light increases perfusion of a skin flap via release of nitric oxide from hemoglobin. Mol Med 2007;13:22-29.

4 Weber MH, Fußgänger-May T, Wolf T: Die intravasale Laserblutbestrahlung - Vorstellung einer neuen Therapiemethode. Dtsch Z Akupunkt 2007;50:12-23.

5 Litscher G: Modernization of traditional acupuncture using multimodal computer-based high-tech methods - recent results of blue laser and teleacupuncture from the Medical University of Graz. J Acupunct Meridian Stud 2009;2:202-209.

6 Litscher G, Xie Z, Wang L, Gaischek I: Blue $405 \mathrm{~nm}$ laser light mediates heart rate - investigations at the acupoint Neiguan (Pe.6) in Chinese adults. North Am J Med Sci 2009;1:226-231.

7 Litscher G: Modernization of acupuncture using high-tech methods at the TCM Research Center Graz. Teleacupuncture bridges science and practice; in Xia Y, Ding GH, Wu GC (eds): Translational Research on Acupuncture. Berlin, Springer, 2010, in press.

8 Rindge D: Lasers and De Qi. Acupuncture Today 2009;10:5. www.acupuncturetoday.com/ mpacms/at/article.php?id=31944.
Yang WZ, Chen JY, Yu JT, Zhou LW: Effects of low power laser irradiation on intracellular calcium and histamine release in RBL-2H3 mast cells. Photochem Photobiol 2007;83:979-984.

10 Enwemeka CS, Williams D, Enwemeka SK, Hollosi S, Yens D: Blue 470-nm light kills methicillin-resistant Staphylococcus aureus (MRSA) in vitro. Photomed Laser Surg 2009;27:221-226.

11 Kushibiki T, Awazu K: Blue laser irradiation enhances extracellular calcification of primary mesenchymal stem cells. Photomed Laser Surg 2009;27:493-498.

12 Kushibiki T, Tajiri T, Ninomiya Y, Awazu K: Chondrogenic mRNA expression in prechondrogenic cells after blue laser irradiation. J Photochem Photobiol B 2010;98:211-215. 